MATT SMITH

University of Portsmouth

\title{
Shifting Modes: Spectatorship, Theatrical Virtual Reality and Motion Capture through the Experience of Fatherland XR
}

\section{ABSTRACT}

This article discusses a project using Extended Reality (XR) within theatre and its effect on audiences who are part of the testing and development of a theatrical production. The article develops knowledge surrounding agency / embodiment and multi-modal story telling utilising VR and motion capture technologies. There is also contained within the article a demonstration of how a University and a theatre company can collaborate using XR technologies. This collaboration is presented based on three interviews with key members of the team. At the time of writing the production is still undergoing final developments. The discussion places the practice within the field of immersive performance and new technologies. Many of the claims made are based on practice based experiences and the messy data provided by test audiences who are asked to freely respond after the showings. The multiplicity of reactions to this performance artwork are discussed in relation to the emergent, accidental and playful results of multi-modal practices often presenting themselves as a set of performative frames instead of a synergistic whole.

\section{KEYWORDS}

XR technologies, agency, embodiment, multi-modal storytelling, immersive theatre. 


\section{INTRODUCTION}

The Creative XR ${ }^{1}$ funded project Fatherland XR involves a team from University of Portsmouth ${ }^{2}$ who are working alongside the Theatre Company Limbik Theatre ${ }^{3}$ using real-time motion capture, virtual reality and audience participation to create live theatrical experiences exploring the themes of dementia and (dis)embodiment. The performance Fatherland XR uses motion capture technology similar to animated avatars in films and projects live scenes through a single virtual reality headset. Various audience volunteers wear the headset, and the rest of the audience sees the volunteer's view, whilst the actor performs around them. The actor wears trackers on their body so they are also part of the projected/animated set. The participant/volunteer interacts with the virtual actor and the live actor on the stage. At different stages of the performance other audience volunteers are asked to take part and engage in the development of the story. One area encountered in this practice is the affective experience of these theatrical digital spaces and the effect on perceived agency for the different modalities of spectatorship.

The narrative in Fatherland $X R$ is presented as a story inside Samuels head and explores how he uses technology to help him share. His fragmented voice speaks from an old cassette player and other sonic devices as well as his mouth. The audience follows him into an animated virtual desert referencing Hollywood westerns and this reminds Samuels of his father and that he wants to tell the audience a story set in an imaginary 'American West'. Early in the exposition there is a break in the narrative when Samuels inhabits the avatar of Wayne, an invented fictional character, who is presented as an angry white American man. Wayne works at Walgreens, lives alone with his incapacitated father and wants to fire Esperanza, their Mexican carer. Frustrated, Wayne concocts his own conspiracy theory about why his father is ill-a theory that involves Mexican witches, giant pharmaceutical companies, and the neighbourhood crows. Fuelled by internet deep dives, Wayne's theory sends all three characters on a journey across California, where Wayne's paranoid Twitter feed collides with migrant farmworkers, $4 \mathrm{G}$ masts and a sacred Native American mountain. Wayne's indignation is a warped reflection of the frustration Samuels feels in the face of his own father's worsening dementia. This emotional turmoil is presented as something that in the real world Samuels struggles to express. Throughout Wayne's story, all three of Samuels's characters interrupt the action encouraging him to confront his own anger, and undertake a journey into the effects of

\footnotetext{
${ }^{1}$ creativexr.co.uk/cohorts/limbik/

${ }^{2}$ www.port.ac.uk/news-events-and-blogs/blogs/xr-events-bring-together-university-and-businesses-from-thecreative-industries

${ }^{3}$ www.limbiktheatre.com
} 
dementia. It's a journey that involves Samuels examining his own ability to be emotionally present amongst the people he loves.

The question considered throughout the project was; how is agency viewed by VR theatre audiences, from both the perspectives of the VR user and the spectator of the VR user? The project explores this by capturing digital immersive audience experiences and one of the most effective methods employed was unstructured interviews recorded on video after the shows. The team also tried asking simple questions as questionnaires and this is presented in this article as a cut up poem that indicates the multifarious responses from audience members. This initial capturing of audiences responses presented here is part of an ongoing project exploring audience engagement with immersive storytelling and explores processes of performance and volumetric capture, sensor-based media, haptics and spatial interfaces. This unique space between the digital and the bodily is presented in relation to audience's experiences throughout the article.

As well as the creative team reflecting the audience considered these issues of agency of 'unbounded' bodies in the performance space. The initial responses were so intriguing that Smith rearticulated them in this version of a cut up poem, inspired by William Burroughs, made from their written responses. These responses Smith re-performed at subsequent research events, ${ }^{4}$ in this way, the audience's feedback had its own performative afterlife.

\section{Audience Feedback Cut Up Poem}

I felt lost, sometimes peaceful, sometimes a bit bewildered. Felt slightly vulnerable.

A spectrum of emotions, feelings and thoughts...

I felt warmer on the stage and in the headset. And a harsh unplugging when it was removed...

It was a guided journey which gave me less agency...

I was simply a vessel in someone else's story...

To be the person the audience can relate to...

I felt less like a part of it and more like a prop with a chance to speak...

As the camera of the story...

I was generally curious and wanted to know more about the story and what was going on...

When I got to investigate I felt more part of the story...

The role of the audiences eyes...

\footnotetext{
${ }^{4}$ One of these events was the Immersive Storytelling Experiences Research Symposium at Liverpool John Moores University. limumediaproduction.com/2018/11/29/immersive-storytelling-experiences-research-symposium/
} 
It seemed to be up to the VR user to make an interesting point of view for the audience... They looked a little clueless...

They froze up...

I felt nervous for them...

Kind of acted as a way into the story for the audience...

Slightly unbalanced and slightly self-conscious...

You are in another person's care...

Disoriented at times. Included. Intrigued. Nurtured dreamlike...

Self-conscious and uncertain, to calm and included, to immersed, to pressurised and selfconscious, to sympathetic and calm...

As part of the project research assistant Dr Bernie Byrnes collaborated in capturing the responses of test audiences through video recorded conversations and simple written questionnaires. Audiences were encouraged to deliberate on 'agency' during the small group interviews and the responses indicate the wide range of affects they felt: 'I loved sometimes that we, the audience, knew much more than the people inside [the VR headset] and the other way around, sometimes the people inside knew more than us' (Recording Transcription C0007). Audiences reacted to the experience of watching the VR user as a central role in the drama, 'It felt like ... the person in the headset was the representative. They represented everybody watching it, so I felt like they went up there and they did their bit on behalf of everybody else' (Recording Transcription 00001). The issues around how watching and engaging across boundaries, that were present in the form of technology and the layers of narrative, seemed to be an important aspect for members of the audience;

...there's like an inside and an outside isn't there? If you're wearing the headset - although I wasn't wearing the headset this time, when you're wearing the headset you have become within the performance. So you have become part of telling the story, even though you don't actually know what the story is... (Recording Transcription 00001).

This unique way a theatre audience could be led through or navigate a narrative presented for one audience member, an exciting possibility, as for them 'it's fascinating that somebody else is controlling your view. Where that 'actor'/participant is choosing to look then that's where the rest of us look ... Like politically, or, you know, to give the audience that much power' (Recording Transcription 00006). This power relation between the audience watching the VR user and the actor within the layers of physically present actions and digitally rendered movements were often centered on the unique practice of the central VR users experience; 'I felt for them because they are vulnerable aren't they? I mean they are sort of being moved around blind, they don't know what the 
hell's going on. I thought that was an interesting texture' (Recording Transcription 00006). This assemblage of textures within the space of the performance could become intensely oneiric, because of the way the audience is placed in relation to the layered narratives; 'It almost feels like you're having a dream at points, like you're creating it yourself even though you know you're not' (Recording Transcription 00021).

In addition to considering agency, groups were asked to consider their experiences of 'participation' and some respondents saw this as an issue that related to their ability to be both active and passive at points in the drama and for one audience member they wanted 'More control ... by the audience over what the headset wearer looked at. The volunteer's first instinct is to explore their environment. This leaves the audience forced to watch their exploration' (Recording Transcription 00021). This problem in the way the performances were set up meant there was a continual oscillation for the audience in their experience of narrative.

I think I was slightly apprehensive because really it's unknown, it's not like a standard theatre performance where you know in advance: these are the seats, stage is over there, it's all hands off... And how's it all going to work...what choices will I have knowing there's to be an interactive element to it. Will I be asked things I don't want to do? ... when you've got the headset on you're thinking 'well should I just be sat here while it's going on around, so it's kind of like being involved in a performance but you're not sure how involved you're supposed to be. (Recording Transcription 00006)

This reaction suggests an unsettling experience of navigating Fatherland $X R$ where no singular position is offered for the audience, instead experience is multiplied and for another respondent it is 'like two lenses: you're already experiencing a double consciousness. You're super aware of this intimate personal experience and it makes you aware of audiences and you come out from the audience' (Recording Transcription C0007). This uncertainty can elicit strong feelings for the audience in relation to the other characters/players within the narrative and the making of the event; 'I feel like what I'm seeing is someone else experience a sensory-memory exercise. So, like, playing with the crows and your putting someone else in that world but I mean watching it, I'm jealous. Like where's my experience here and I'm part of your crew, I'm not part of the narrative, I'm not a character' (Recording Transcription C0006). For audience members with limited experience of VR this immersive environment was not altogether a positive visceral experience;

I had the headset on for a while and I've never ever done any VR before so I found it quite weird. And I felt a bit sick and a bit disturbed by it, I didn't really enjoy - it was very interesting and unusual, but I definitely felt a bit freaked out by it, by being in there and I 
did, when it kind of clamped onto my head I had to breathe quite a bit to sort of - and also just the being on stage and having that sort of...I didn't want to do it really, but I, the ball's been thrown to me, but then, I'm really glad I did it because it was a really interesting and great experience... (Recording Transcription C0007).

This interaction and experience of a new format also matches the interests and experiences of a more general theatre audience reacting to the theme of dementia as culturally prescient and one respondent positively reacted to this theme; 'what I thought was terrific about being inside is that it really gives you the experience of somebody or something maybe close to the experience of having dementia, or being disempowered, or...that aspect of being immersed in a world where you, where you know, your power has gone...' (Recording Transcription 00006). These responses are a glimpse of the recorded sessions and indicate the richness of the project, but also the depth of what can be developed through open conversations with audiences. It often felt as researchers a precarious position eliciting these responses, as well as exciting role in encouraging audiences to feedback in this way. This method also indicated courage on behalf of the creative team to allow their artistic process to be scrutinised in this way.

These responses indicate that when employing technologies and the body this can produce immersive responses in the audiences and provokes them to consider augmented presence beyond its normative boundaries. A visionary from the sixties who ghosts attempts at exploring this interface between technologies and the body is Marshall McLuhan. His idea of how the developments in electric technologies immerses the body and extends its network of possibilities (McLuhan and Fiore 1997: 35) chimes with the intentions of the Fatherland XR explorations into embodiment and performance augmented by motion capture and VR. In this experience of watching VR as a spectator, the way the body is extended through new technologies is articulated. This unbounded body is an exciting possibility for the audience and a recurring performance trope. The way this use of technology changes temporalities in the performance space is a reflection of the wider context of how digital technologies have changed lives and our experience of the everyday. The quotidian experience translates back through the constructed reality of Fatherland $X R$ and at some points in interludes, for example, when Samuels asks audiences to tweet with the avatar character of Wayne. This produces a breaking through of boundaries between the digital and the fleshy, partly framed through the emotional core of Samuels's personal story rendered through complex constructions of simulated environments involving invented digital characters like Wayne. This space produces an effect on the body that relates to McLuhan's ideas about the radical nature of technologies. 
One of the peculiarities of an electric technology is that it speeds up this process of transformation. Instant and total rehearsal of all past and all processes enables us to perceive the function of such perceptual returns as one of purgation and purification, translating the whole world into a work of art. (1997: 183)

This possibility of transformation is certainly in evidence in the performances of Fatherland $X R$ and as the narrative deals with memory and dementia the issue of 'perceptual returns' is heightened in the world of the performance. Through storytelling, Samuels testifies and conjures the presence of his father through immersive technologies and the artists invite the audience into this space of 'fractured' catharsis. The emotional core of the father mixed with technology paradoxically both alienates the spectator from the ritual purgation of performance as well as heightening the emotion of this affective experience. This is a novel way of exploring the inhuman problem of new technologies and their future developments.

Jean-François Lyotard toward the end of his life troubled by the problem of the inhuman through the acceleration of technology developed a critical response to the dystopic possibilities of advanced capitalist futures (1991). For Lyotard we moved beyond the posthuman towards the inhuman due to the way that technologies of the fin de siècle ruptured the ability to uphold humanities position in regards to simulations and layers of actualities experienced by the northern hemisphere (Sim 2001: 15). Conversely, the problem of the cyborg body is one that is valorised by Donna Haraway. For Haraway the mix up of technology and bodies in the figure of the cyborg is 'about transgressed boundaries, potent fusions, and dangerous possibilities' (1991: 154). In Fatherland XR the embracing of technology means that the issues of the inhuman are brought into the performance space and this troubles narratives of human nature and thought within the complex space of multi layered viewpoints. Haraway celebrates the inhuman in her concept of the cyborg and this concept is represented through the cyborg performance of Samuels in Fatherland XR and his obvious use of motion capture suit rendered within a projected digital environment. This performance is not celebrating the machine, it instead indicates through the use of embodiment of digital technologies that the 'machine is us' (Haraway 1991: 180) and this cyborg performance can be performed in a theatrical encounter. This encounter with cyborg forms of embodiment can be enjoyable for an audience and can produce positive aesthetic experiences.

Intense pleasure in skill, machine skill, ceases to be a sin, but an aspect of embodiment. The machine is not an it to be animated, worshipped, and dominated. The machine is us, our processes, an aspect of our embodiment. (Haraway 1991: 180) 
Samuels performs this machine skill with the rest of the ensemble, both human and inhuman elements in the performance space. The audience are invited into this world of multiple layers of embodiment through different levels of involvement, either with or without the 'mask' of the VR headset.

The role of the audience member in Fatherland $X R$ is certainly not passive even for the main body of the audience who watch the interactions between Samuels, the VR user and the shifting immersive landscape. The audience in this space are what Jacques Rancière (2009) suggests is an 'emancipated spectator' given the possibility to engage in the work of art actively. The spectators inhabit 'the role of active interpreters, who develop their own translation in order to appropriate the story' (Rancière 2009: 22). Within the world of Fatherland XR opportunities are built within the dramaturgy to allow this space where the audience can stitch together narrative, visual and affective information into their own story. This approach to the audience allows them to be 'both distant spectators and active interpreters of the spectacle offered to them' (Rancière 2009: 13). Rancière questions the problem of the stultifying nature of the 'hybridisation of artistic means appropriate to the postmodern reality of a constant exchange of role and identities, the real and the virtual, the organic and mechanical and information based prostheses'(21). Rancière suggest we move beyond this position in performance cultures through the role of active audiences who have the ability to 'problematize the cause effect relationship' (22) in theatre through active involvement. It certainly was a clear aim of Limbik to have a very active audience, to the extent that much of the process involves the audiences making the work, for example, the audience testing process throughout became central to the praxis. Capturing this complexity for the researchers is also one of the challenges of this process.

Fatherland $X R$ is developing an emergent form of theatre exploring technologies; but it is also exploring theatrical issues that have a lengthy genealogy. The issues in the devising around how to work with audiences who are viscerally immersed and then alienated through the limitations of the technology have been explored over the last one hundred years by practitioners and visionaries like Brecht and Artaud. Rancière (2009) reworks these practitioners into his ideal of the 'emancipated spectator' as a relevant way of thinking of the radical possibilities of positioning audiences in regards to theatre. In the process of Fatherland XR the legacies of twentieth centuries avant-garde has effected the aims of the company to clearly involve the audience as both developers and active makers of the work. The audience is also very sophisticated in the process of developing Fatherland $X R$ as they are often invited from university or arts field contexts and so are well aware of self-referential playful genres and ironic forms that are found within popular culture. Treating the audience as 'emancipated spectators' during the early stages of the development also meant that 
they are co-creators and researchers into the possibilities of this form. It seems that if the audience are not just receivers but also producers then this is one of the radical potential outcomes of the Fatherland XR experiment. Even though a great deal of varied responses were recorded, it remains clear at this stage that many questions about the use of these technologies in performance remain unanswered and need further interrogation.

Helen Freshwater (2009) suggests that the methodology of researching theatre audiences falls into two clear domains, the first method is conducted for the use of marketing and uses a mix of qualitative and quantative data to profile audiences and demographics. The other kind is the messy but often deeper method of capturing audiences experiences based on discussions and interviews. This use of simply asking the audience 'what they make of the theatre they see' leads to insights into the diversity of responses to shows (2009: 33). This attitude towards capturing the diversity of responses inspired the aims and intentions of the Fatherland $X R$ audience testing. After the performances the unstructured conversations encouraged by researchers led to dynamic and complex comments about what kind of performance the audience had just witnessed. These conversations captured on video were then part of the development of the performance. Samuels repeatedly suggested that the audience are developing the work with Limbik in these events throughout the devising period. This open process does put particular demands on the makers of the work as they engage with the range of responses (including negative comments) offered by the audiences invited to the testing phases.

The way audiences receive multimedia live art performances is presented by Iryna Kuksa (2009) as a complex relationship where the issues of simulation and representation are pertinent to understanding audience's experiences. Kuksa suggests that 'multimedia technologies and virtual reality often serve as a means to help the spectators sacrifice realism for the sake of the live art experience' (93). She further posits that a distinction should be made between multimedia art producing representations and VR producing simulations (93). In the case of Fatherland XR the issues of representation and simulation are definitely unresolved in the period of devising the work with audiences. The limitations of immersive technologies means that the participants with or without VR headset intentionally 'suspends disbelief' ${ }^{5}$ if they wish to develop a deeper experience of the performance. Although the performance relates to a real referent, in this case Samuels's own personal biography, the way the performance space is set up means that the performance is a distinct departure from stage realism. To perform in the show the actor Samuels also employs physical theatre and mime techniques which are anti-realistic and precede naturalism but are rooted

\footnotetext{
${ }^{5}$ Samuel Taylor Coleridge
} 
in the history of theatre genealogies. This is further stylised and 'made strange' (Brecht 1978) through the way the majority of the audience are witnessing the VR experience from a relatively distanced position as spectators. The story being told in Fatherland XR holds the disparate levels of experience together to some extent in the performance, but the audience are active in the way they are viewing and participating with a simulation of an actuality in front of them. This physical participation is combined with feelings of empathy shared for actor and VR user interacting within the performance space.

Kuksa (2009) offers the notion of the 'anti-theatrical' (94) nature of VR technologies and in developing the way the performances evolved Samuels and his team seem to be aware of the limitations of this technology within the theatrical frame. In their endeavour they sought the possibility of a new genre of theatre that adopts a novel way of thinking and appropriating discourse about how digital technologies can function in the ephemeral space of performance. In the stage of audience testing and devising it appears that VR and motion capture presented as theatre has the possibility of both immersing, interrupting and alienating the audience from the work. This happens through the accidental events when the image or the technology corrupts or breaks down, or when a seamless shift between spaces occurs. When avatars move out of a room incorrectly or an arm pushes through a wall it clearly shocks the audience and interrupts the simulation, but it also bends stage reality towards surrealism, like within a Jean Cocteau film. ${ }^{6}$ Interruption caused by technology generated comments from the audience as a problem in disrupting their feelings of immersion within the VR environment from both the perspective of the VR users and the spectators viewing participants. Limbik are open to the possibilities of the technology disrupting the space and 'making strange' the way the piece is received. This strange imaginative shift for the audience to participate in the space of Fatherland XR means there is a reflexive relationship that develops between the simulation and its theatrical context. Limbik are navigating this difficult terrain, one in which for Kuksa 'It is difficult to control the work of art placed in the virtual space - from how it was created to how or should be perceived by the audience '(95). This undecidability of the artwork, when using technologies, opens up a space where the audience both question the perceived augmented realities experienced and additionally chooses to imaginatively play with these possibilities and limitations within this new genre of performance.

Giannachi (2004) suggests that in her definition of VR theatre this type of performance 'remediates not only the media, but also the viewer's performance' (8) and this relationship with the

\footnotetext{
${ }^{6}$ Beauty and the Beast (1946)
} 
viewer is one that is active and engages them within the spectacle produced through many layers. As well as the viewer being active in the artwork the viewer for Giannachi is 'inside the image' (9) of VR theatre and this theatre has the capacity to disperse and multiply the point of views for the audience. By entering into the experience in this way the audience for XR performance are entering into the work of art both in control of the space and within the picture (11). This position of the audience can be emancipatory but also one that creates a sense of dislocation as opposed to location within the rhizomic fragments of the bewildering VR theatre experience (11). This process produces doubt about the state of the subject in relation to the work and figures appear 'out of joint', doubled, bodies becoming 'spectral' figures moving and navigating the space of VR theatre. Pioneering scholars of the interface between immersive theatre and live art, Gabriella Giannachi and Nick Kaye (2011) explore the issues of presence within VR, through collaborative experiments with theatre practitioners, using the immersive environment of a CAVE (Computer Automatic Virtual Environment) set up, using a SGI (Silicon Graphics Incorporated) computer systems, developed by Professor Mel Slater at University College London. The CAVE project developed by Kaye and Giannachi involves studying a cube of projected interactive images that simulate 3D experience and the interplay of real bodies performing within this cube and the telepresence of the bodies in VR. The experiments and research as part of CAVE develops a viewpoint that presents the subjects as entering a 'palimpsestual relationship' (89) with the intertextual signs of VR immersion. This can be a 'disjunctive experience' involving the 'simulated and the real, provoking an awareness of the layers and doubling' (91). For Kaye and Giannachi there is a 'dissonant relationship' (92) between the virtual and the actual bodies and objects within the immersive space of CAVE. This uncertainty between spatial presences troubled by VR is heightened within the space of theatrical framing and theatre practice does not 'close the gaps' between these layers of (un)realities. In VR theatre this fracturing between modalities and presences opens a reflexive space within which audiences/participants/users can question and reconsider the augmentation of the corporeal in digital spaces. This interstitial space of uncertainties about presences, is a feature and playful space within the performance frames of Fatherland $X R$, which at times is both exciting and disconcerting for artists and audiences.

Charlton and Moar (2018) have recently explored the possibilities and the ruptures that the mingling of the virtual and the dramatic produce. They valorise the use of immersive technology to render theatre as a 'dialogic exchange' (192) between elements of the mise-en-scène. Within this dynamic space the proxemics that VR immersion offers is mixed with the sense of 'scopophilic voyeurism' and 'ludic possibilities' (193). They posit that these experiences can be both 'personal and oneiric' (194) for participants in the process and performance of XR. They see that this type of 
practice 'offers another medium in which we can get up close and personal with human beings in their moments of soul searching, emotional turmoil, terror and joy'(194). Charlton and Moar relate that the ludic voyeurism in VR immersion is a place in which 'touch 'can still be evoked and actually encouraged' (196). Touch became an important consideration in the way the actor and the participant enact 'care' in the Fatherland XR experiments. Within Fatherland XR the playful environment set up by the company provided a method to immerse the audience within a platform that was connected to 'real' personal experiences of Samuels's biography. Machon (2013) suggests that the immersive environment using performance and digital enhancement offers the possibility of a sensual (35) engagement with an active audience. It was the intention of Fatherland XR to sensually touch the spectators by engaging them physically on multiple layers and inviting them into the touching story of Samuels's father. Sensual touch through the interface of digital performance is a theme that connects pertinently in the project to; (dis)embodiment, haptic interfaces, liminal spaces of digital performances, environmental affects and agency.

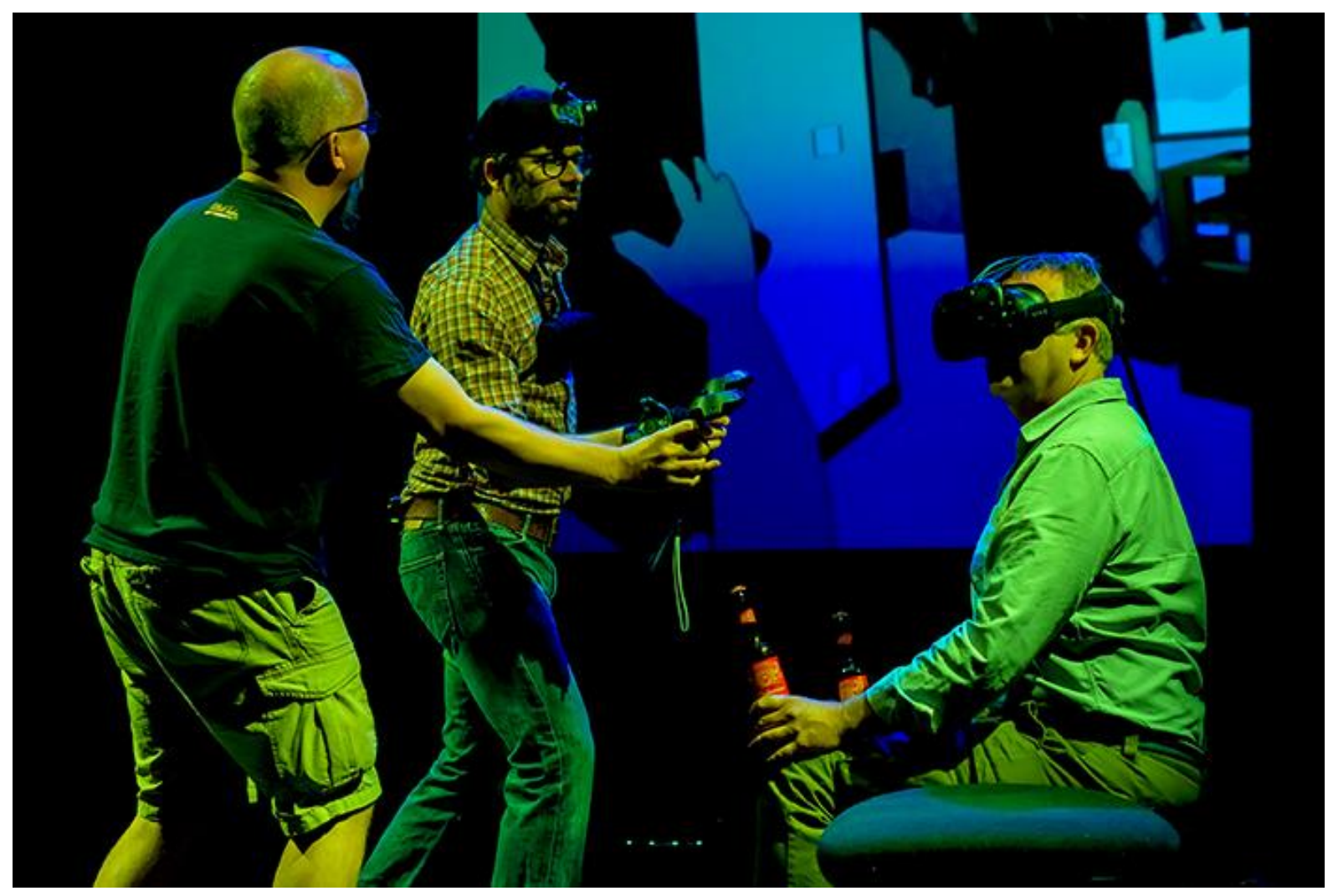

Figure 1. Alex Counsell, Ben Samuels and audience member exploring use of beer bottle props and trackers. Photograph Limbik. 2017

\section{DEMENTIA AND DISEMBODIMENT}

A central part of Fatherland $X R$ is the personal story of Samuels's father and his experience of dementia played out throughout the scenarios in the performance. The perceived change in sensory 
experience through inter-medial forms represents a fracturing of this experience that distorts the character of the father. The father becomes distorted through the way the digital does not fully represent a tidy identity or a fixed space of experience. The digital experience of the VR headset troubles augmented reality in this unique theatrical space of (dis)embodiment. This is witnessed by the main body of the audience not wearing VR Headsets. The use of motion capture represents another metaphor of (dis)embodiment when Samuels, at the end of the performance, removes the sensors and the projected avatar is disrupted and broken. Although not exactly describing the pathology of dementia this use of the digital space in Fatherland XR opens spaces in which to reconsider mental impairment and illnesses through a simulated space. This theatre presents new metaphors for illness, through storytelling using immersive technology, by heightening the issues of embodiment and fractured experience for the active audience.

One of the intentions of Fatherland $X R$ is to produce affect in the audience by eliciting the real referent of Samuels's relationship with his father within the augmentation and digital rendering. This affect is then represented when the VR user is cared for by the performer. This caring relationship between the VR user and performer roles indicates to the audience that the VR user's agency appears dependent and diminished within the space. This relationship and reduced agency reflects the experience of Samuels's father. This is particularly moving, in one key scene, when the VR user mirrors the performer's movements. This use of the mirror metaphor signifies shifting identities in both the characters, their rendering as avatars, the relation between real versions of stories and the audiences constructed narratives. This mimetic disruption complicates the shifting modalities of the audience because they can choose to watch Samuels in motion capture suit, his projection as avatar, his avatar through the VR headset projected, the VR users experience through their body movements, the controller of the operating systems etc. Overall the performance presents a set of modes that are complex to capture and in the feedback from the audience they often described unique experiences of navigating this complex space. 


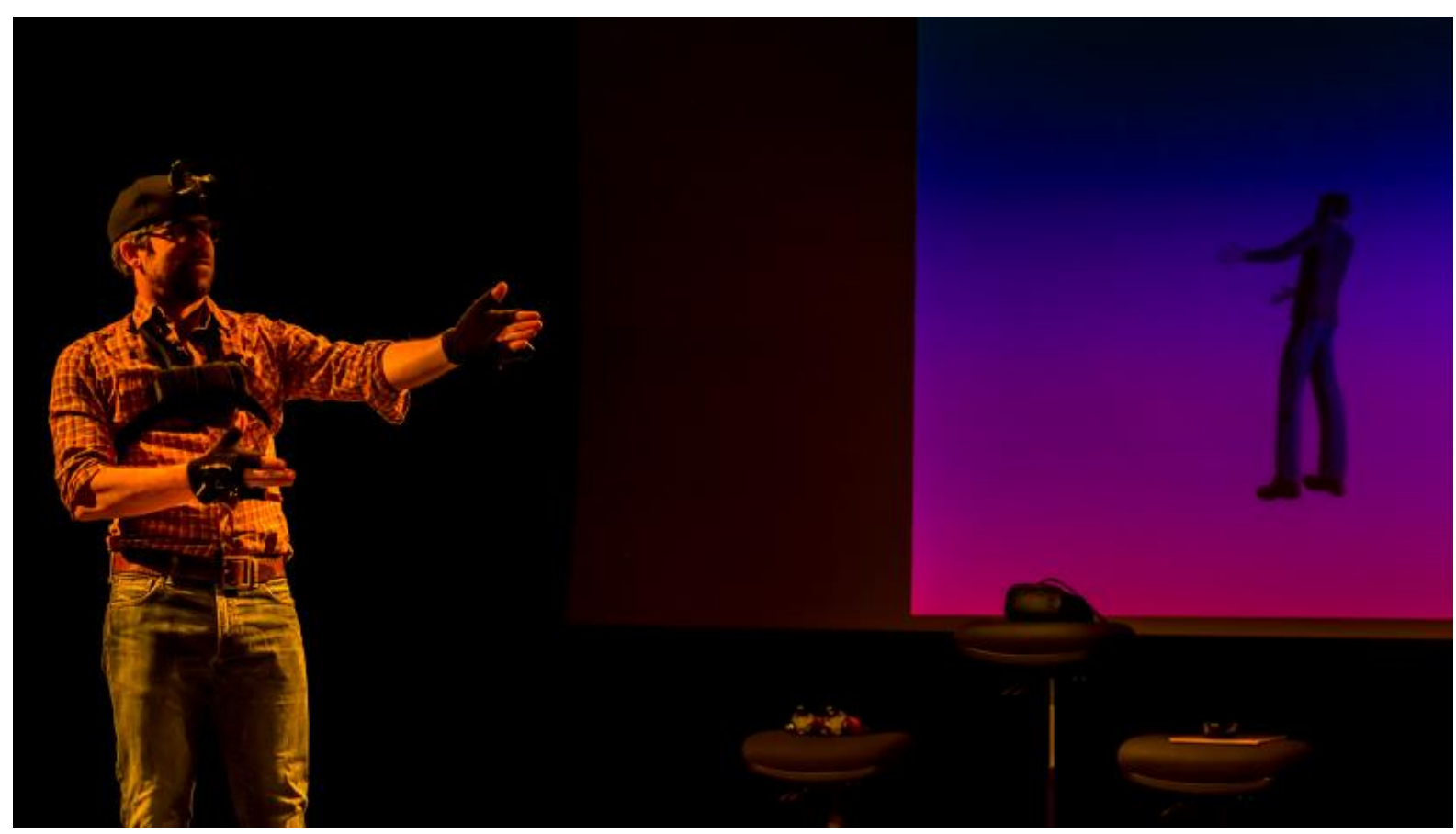

Figure 2. Ben Samuels and avatar during early development phase. Photograph Limbik. 2017

During the show the use of props, media archaeology and touch are all central to the way the performance works within the space. The emphasis on the haptic experience of the VR user and the audience as witnesses in this performance suggests and evokes the touch of the actual and the representation of memory. Samuels records instructions on cassette tapes and plays vinyl records as a way to memorialise objects in the surrounding space of motion capture and VR. The clink of real bottles attached to motion sensors enhances their value and effect in the performance space. Samuels also touches and guides through touch the volunteers from the audience and this touch is clearly developing and changing the experience of the VR user, but also the way the audience can choose to read the event. Without this touch, or care through touch, the event would feel alienating and distanced. The audiences expressed a desire to touch or be touched through the hybrid forms and multiplicity of signs that forms the experiences augmented by technology in this performance. This careful touch of the performers hand is both a guide in navigating this complex space and part of the haptic experience.

Fatherland $X R$ operates within in an interstitial format and its plurality of undefined form makes it a complex and intriguing performance event to experience. It constructs an environment that is imaginative but also lost in the spaces between experiences where embodiment can be troubled by actual and virtual objects, sensory experience and the incommensurable experience of 
an 'others' mental state. Throughout the piece the digital and the bodily are not unified into a complete gesamtkunstwerk, but are within a fragmentary space where presence and agency blur in an out of focus. This space is a liminal space where the audience is a co-creator of meaning and where the mixing of media both interrupts and in a reflexive way speaks to the audience about the issues of real and constructed narratives within the immersive world of digitally augmented performances. Conversations from key members of the development team explored this process of making immersive performance in relation to questions of agency, reception and shifting modes as they developed as part of this project. In these conversations, the artistic team was provoked to reconsider the audience's role and function within this process.

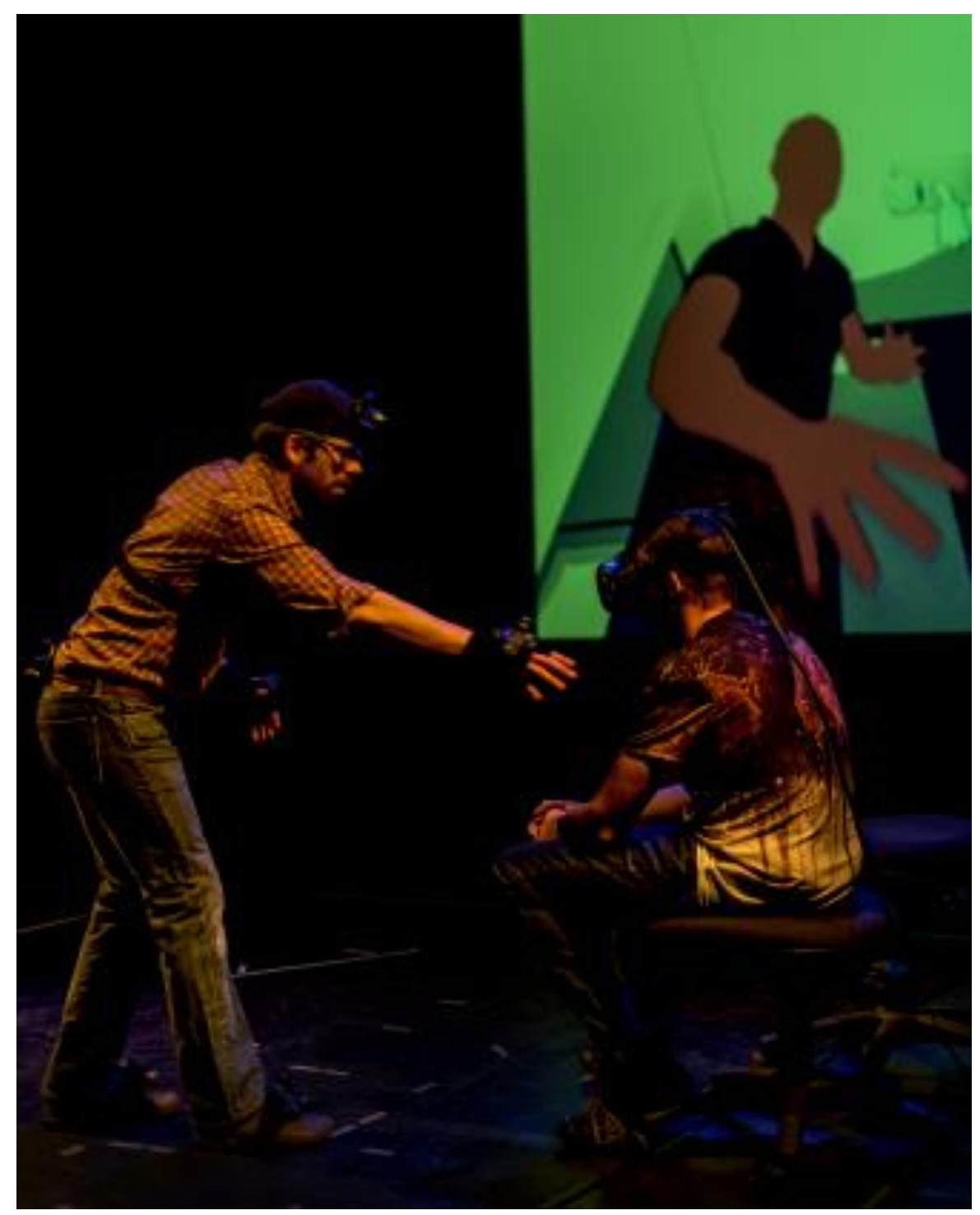

Figure 3. Ben Samuels and audience member. Photograph Limbik. 2017 


\section{CONVERSATIONS}

During the development of Fatherland XR interviews were conducted with key members of the creative team with the focus of the discussion around the way the audience are involved in the process. The producer Lou Doye ${ }^{7}$ expresses her interest in the way agency for the audience can shift due to the use of technology and how there is potential for the audience participant onstage to radically change; The person in the VR headset controls and captures images viewed by the rest of the audience, becoming a film director.' For Doye this is not an experience that is the same for all audiences and that she believes Younger gaming audience members were less likely to enter the stage VR environment but once they did, [they] had a stronger sense of their surroundings and were more likely to walk around the space more and experiment with the viewpoint of the VR character they were embodying.' The challenges of this project meant that Doye had to adapt her approach as the producer in this new medium needs to gain an understanding of the timelines of both the theatre and gaming art forms...communication with all creatives and the translation of language between both art forms helps to evolve the project '. It is a general point, made by Doye that the creative team had to open out their practices to the experimental multi modal formats adopted in this project. This was further complicated by the practical issues of using complex equipment in different venues; 'Further learning on the tour-ability of real-time motion capture kit has been a challenge and the ability to merge design such as lighting and sound in balance with the motion capture kit has been challenging.' Doye suggests that these new methods will become more embedded in the future as the more we cultivate the technological and theatrical collaborations the more products will be on the market, hence audiences will become more familiar with the experiences, and venues will seek to programme such work'. Agency and embodiment are complicated for Doye as she perceives the audience watching the actor and participant onstage as framed in a particular way; 'It is a little voyeuristic for the audience watching, anticipation of the actions of the headset wearer and the view they take becomes exciting, as their view takes the film directors role...the person in the VR headset naturally takes on the body physicality of the character they are embodying in the headset, whether that is a frail older person or the middle-aged irritated man.' This change in embodiment means that the audience feels the VR wearer oscillates in agency within the onstage environment. 'The interaction between the actor and VR wearer is integral to the concept of the performance...I feel the VR wearer's presence increases.' Overall, Doye is suggesting that in developing this work the audience is part of the collaboration and co-creates the development of new technologies in performance during the test events and after the showings

\footnotetext{
${ }^{7}$ www.loudoye.com
} 
when giving feedback; 'Audiences are integral to the development of live theatre with XR technology. To understand the mechanics that work and compliment performance we need to consult with the viewers from all demographics.'

Alex Counsell VR developer and Technology Producer, similarly to Doye, emphasises the novel way this production developed; 'Fatherland XR is unique in this context, as it has been developed primarily as a theatre work and not a VR work... If we continue to explore theatre and the way that it can work with this technology then I think a new medium is being created'. Counsell suggests that one key artistic choice involved making the use of technology, computers, motion capture cameras and trackers visibly present within the staging, this foregrounded these elements within the artificial theatrical frame, 'by fully integrating the tech into the play, which was a creative choice made by Samuels...'. The theatrical frame also presented its own set of challenges involving what was conceivable and possible and also finding potential in the unforeseen; 'I think that the compromises are the most interesting area, as this is where new ideas are formed, and then they inform the rest of the process... whilst trying to find a solution we stumble across a new idea that may have come from a glitch or error, but works'. Counsell also highlights the way that watching VR immersion offers aesthetic pleasure; 'I suppose I just assume that the people wearing the VR headset will be immersed and entertained as this is the medium that I know best, but what has surprised me is just how much people enjoy watching from the seats. My favourite quote that I overheard someone say was that "...this is the first time I have actually enjoyed watching someone do VR...". Counsell, beyond his role as designer of the technology, observes the affective response in himself and the audiences when he suggests that he 'felt empathy towards the wearer when they show an emotional response, especially when there is someone they know, who has suffered with dementia and [they] connect with that through the technology.' During his observations Counsell considered that there were distinct types of response to the role of VR user when they are participating in the stage environment;

We have come up with terms to describe them; 'Walkers' - people who are not afraid to get up and walk around on stage and need constant attention from Samuels and are capable of breaking the narrative or scene that we have prepared with unpredictable behaviour. 'Talkers' - These people like to vocalise the virtual part they are playing and happily talk back to Samuel when prompted or not. 'Statues' - The ones that sit still and hardly move through fear of the tech or being on stage, this has the possibility to affect what the audience see through the headset, meaning Samuels has to encourage them to do more...Depending on their stage persona, this affects their presence for the audience watching, and as the play is immersive and in VR for the wearer, it would affect their 
presence or agency in the virtual scene. By being more active and engaged their presence for Samuels is affected also... it works on a few levels...

Ben Samuels, the solo actor in Fatherland XR and Artistic Director of Limbik Theatre discusses his relationship to the audience member in the VR Headset as one of care and trust which mirrors the themes around his relationship to his father and his father's dementia. 'My first impulse to the volunteer once they are in Virtual Reality is to make sure that they feel safe. Next, it is to guide them through the story.' When this is established ludic possibilities open up between Samuels and the audience member within the story; 'Once I have a sense of how they react and respond to the world of the story, I begin to play with, and off of, their instinctive responses.' Within this role Samuels becomes both actor, narrator and carer for the onstage audience member and this relationship is extended beyond this relationship towards the audience who witness the story from outside of the stage space.

I see myself as a storyteller, a storyteller using technology. To the volunteer, and by extension the audience, I feel that I am both guide and trickster, and in order to be the trickster they have to trust me first. That trust is built from the moment I 'select' them to come onstage, by catching their eyes and throwing them a ball. The process of building trust starts with that eye contact. It continues by making physical contact with them before they put the headset on. Shaking their hand, touching their shoulder, familiarising the volunteer with the sensation of contact. As well as asking them to do simple tasks-sitting in a chair, standing up - tasks that will repeat, and become much more difficult, once they have the headset on.

This multi-modal format of personas and roles for Samuels as actor presents a unique power relation between his presence and the audience; 'I actually feel quite vulnerable during the performance, because I am at the mercy of so many variables outside of my control-the volunteer, the technology...I feel this vulnerability brings a kind of calm, a relaxation as the performance settles into its rhythm, and my attention really falls on my interaction with the volunteer. My focus is on and with them.' This focus on the audience participant is fractured through the lens of the layered technologies within the mise-en-scène and within the environment set up by the company. This becomes an immersive experience, with the relationship between Samuels and the VR user at the heart of the drama; 'the VR headset effectively achieves that same result... It immerses the volunteer in the world of the story, and they forget they are being watched... If I spend too much time 'winking' at the audience, because I am essentially onstage with a volunteer who is blind to the physical world around them, I lose their trust'. The participation of the audience is not fixed in this 
relationship dynamic but is complicated and enhanced through the interface of digital technologies. This involves layered narratives that Samuels develops through the process when working with new audiences. Within this site of performance and its use of media frames Samuels employs methods to navigate the trust and care needed between participants, which then establishes a collaborative rapport with the audience; 'I can feel them relax, as the ground rules of the show are established and re-enforced as they play out... I meet them with a mixture of curiosity and suspicion'. This new theatrical format has as its referent film cultures, both in content and form and this Samuels describes as the virtue of this project as 'at its; heart, Fatherland XR is storytelling. But storytelling that uses highly sophisticated technology and audience volunteers to become a kind of live film. A virtual reality film created live in front of an audience's eyes'. In this dynamic relationship between forms, live theatre troubles the film referents in Fatherland XR. The live actions purposely provoke questions about the establishment of fixed identities in relation to both the narrative content of dementia and the formal artistic decisions enhanced with the use of technology.

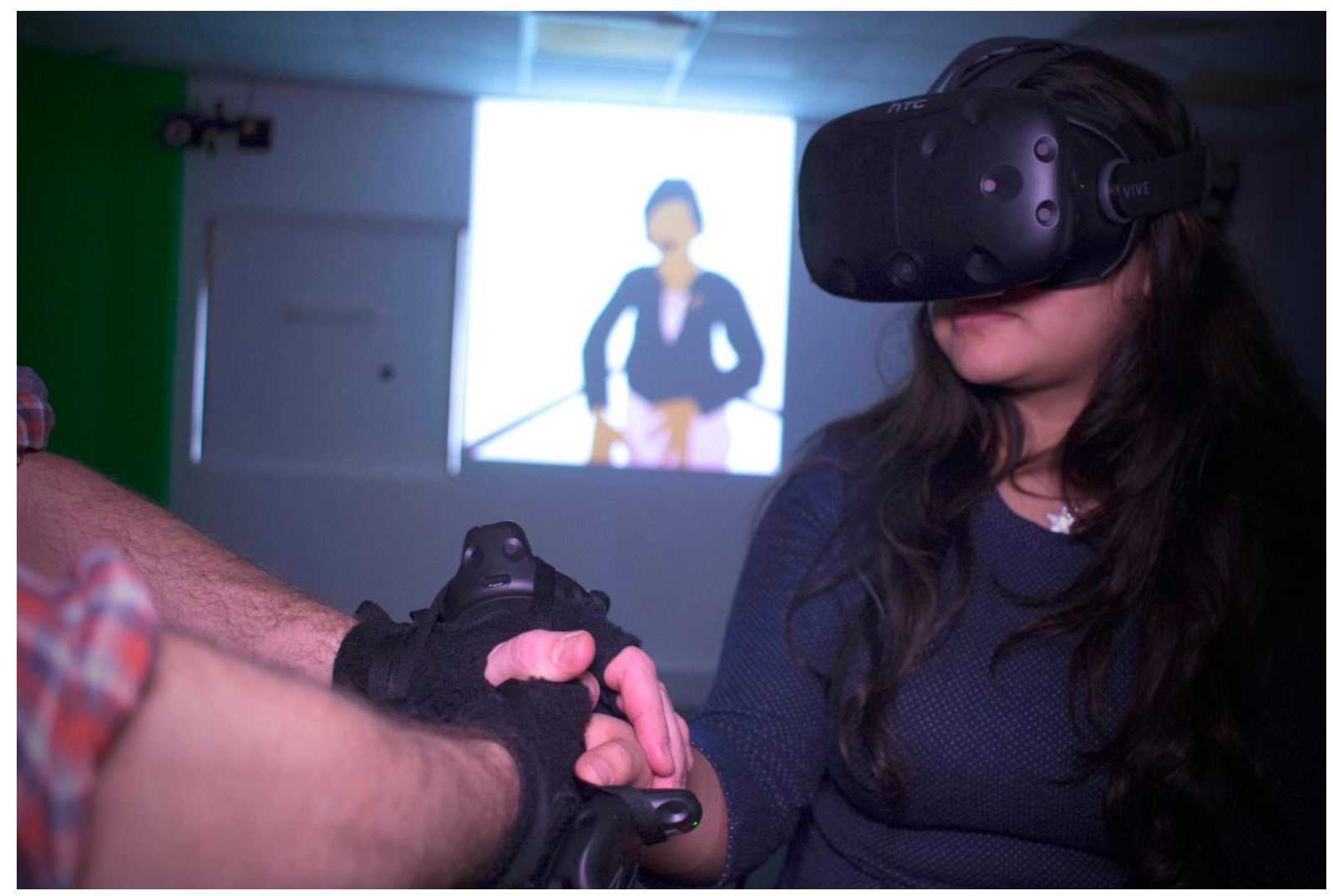

Figure 4. Ben Samuels and audience member exploring touch. Photograph Limbik. 2017 


\section{CONCLUSIONS}

Through these conversations with artists and audiences, the issues of how artists and researchers include an audience in developing new modes of performance are complex and often interruptive to a process that involves a collaborative team accepting the unpredictability of live environments. Creating work with audience testing at its heart means that the devising is reactive and uncertain. The interface between theatre and digital media produces a terrain that involves an open process and layered texts within which artists navigate with participants. Drawing together the most prescient threads of the conversations with some of the creative team, it is clear that Fatherland $X R$ is a live film that uses the primacy of storytelling and theatre. Timelines are described as different in this type of process for the collaborative team when temporalities are stretched because of programming and the development of XR environments. The use of technology plays a supporting role and this indicates a possible way that theatre can work with the digital. Suitable alternatives are often necessary in the process of making in this flexible and multi-layered manner. The storyteller as trickster is one role that actors can adopt within this mixture of forms, when bringing together disparate elements that do not synergistically fit together in the improvised space of performance. Throughout the process, the development of trust between audience and actor is integral to the effectiveness of the performances.

Film audiences and gaming audiences present different modes and ways of being within the digital theatre environment in this type of project. Pre-conceptions of an audience always play a role in how the work progresses and the journey is encountered. Reluctance to engage by audiences has to be understood when testing on diverse audiences and anticipated by the creative teams. Volunteer immersion and the journey of the audience involves empathy, mixed with enjoyment and voyeurism, based on the experience of audiences and the creative team. This aesthetic can be created if the participants if they are nurtured through safety and guidance in the ludic space of theatre and XR. Participation is a central element to the development and embodiment is key to the theatre XR aesthetic.

It is clear that the audience involvement is unique. Unique audience experience is viewed as integral to both the making and presentation of the work and the audiences knowledge acts as a proof of the artistic experiment. This engagement with film, gaming and 'traditional' theatre audiences using new technologies represents new experiences of imaginative performance environments. Overall, through conversations it is generally apparent that curiosity and suspicion are part of the audience's journey. 
Adapting models and ways of engaging is a continual thread in the way the team conducted the project. Merging design elements within this process can be challenging, but has to be recognised as part of the process. Merging theatre and motion capture elements involves compromises and serendipity. This accidental knowledge can become the seeds of the artwork. Even though the work is often experimental the desire to entertain is still often an aim of the team of makers in this example. Rehearsing 'by doing' the show is inherently risky in this format but in this case was the method adopted. Interaction and improvisation develop the theatrical space and also effected the increased presence and the different levels of 'being there' including role playing. Throughout the performance there is a sense of both disappearing and being larger than life, for all the bodies involved in the mise en scène. This mixing of layers of imagined and embodied realities forms a heady mix of entanglement as expressed by Denise Doyle when she defines the transformative effects when performance and digital mixes; 'As the real and imagined are no longer strangers (or opposites), it is also true that the physical and the virtual have become more firmly entangled.' (2016: 33).

The modes of being for both the audience and the performers in Fatherland XR is never fixed and is constantly under revision and often provisional in the space of both the making and the performance. Digital technologies make this experience more pronounced and challenging. Fixing and capturing are questionable, as the theatrical landscape constantly shifts the way the technology can be applied. This incommensurable experience has to be valorised otherwise it becomes a continual problem of glitches and interruptions for the makers, participants and witnesses. 


\section{REFERENCES}

Brecht, Berthold, \& Willett, John (1978). Brecht on Theatre : The Development of an Aesthetic. London: Methuen.

Charlton, James Martin \& Moar, Magnus (2018). 'VR and the Dramatic Theatre: Are They Fellow Creatures?. International Journal of Performance Arts and Digital Media, 14(2), 187-198.

Doyle, Denise (2016). 'Travel, Space and Transformation.' Metaverse Creativity (new title: Virtual Creativity), 6(1-2), 25-36. Bristol: Intellect.

Freshwater, Helen (2009). Theatre \& Audience. Basingstoke: Palgrave Macmillan.

Giannachi, Gabriella (2004). Virtual Theatres: an Introduction. London: Routledge.

Haraway, Donna (1991). Simians, Cyborgs, and Women: The Reinvention of Nature. London: Routledge.

Kaye, Nick, \& Giannachi, Gabriella. (2011). 'Acts of Presence: Performance, Mediation, Virtual Reality.' TDR/The Drama Review, 55(4), 88-95.

Kuksa, Iryna (2009) 'Cultural Use of Cyberspace: Paradigms of Digital Reality.' In Oddey, A. \& White, C. A. (2009). Modes of Spectating. Bristol: Intellect.

Lyotard, Jean-François (1991). The Inhuman: Reflections on Time. Cambridge: Polity Press.

Machon, J. (2013). Immersive Theatres: Intimacy and Immediacy in Contemporary Performance. Basingstoke: Palgrave Macmillan.

McLuhan, Marshall, Fiore, Quentin \& Agel, Jerome (1997). War and Peace in the Global Village. San Francisco: HardWired.

Rancière, Jaques (2014). The Emancipated Spectator. London: Verso Books.

Sim, Stuart (2001). Lyotard and the Inhuman. Cambridge: Totem Books. 